\title{
Pure strategy equilibria in games with private and public information
}

\author{
Haifeng $\mathrm{Fu}^{\mathrm{a}}$, Yeneng Sun ${ }^{\mathrm{b}, \mathrm{c}, *}$, Nicholas C. Yannelis ${ }^{\mathrm{d}}$, Zhixiang Zhang ${ }^{\mathrm{e}}$ \\ ${ }^{a}$ Department of Statistics and Applied Probability, National University of Singapore, \\ 6 Science Drive 2, Singapore 117546, Singapore \\ ${ }^{\mathrm{b}}$ Department of Economics, National University of Singapore, 1 Arts Link, Singapore 117570, Singapore \\ ${ }^{\mathrm{c}}$ Department of Mathematics, National University of Singapore, 2 Science Drive 2, Singapore 117543, Singapore \\ ${ }^{\mathrm{d}}$ Department of Economics, University of Illinois at Urbana Champaign, Illinois 61820, USA \\ e Department of Economics, Johns Hopkins University, 3400 North Charles Street, Baltimore, MD 21218, USA
}

Received 25 March 2006; accepted 6 May 2006

Available online 27 June 2006

\begin{abstract}
We introduce a new game form which allows the players' strategies to depend on their strategy-relevant private information as well as on some publicly announced information. The players' payoffs depend on their own payoff-relevant private information and some payoff-relevant common information. Under the assumption that the players' strategy-relevant private information is diffuse and their private information is conditionally independent given the public and payoff-relevant common information, we prove the existence of a pure strategy equilibrium for such a game by developing a distribution theory of correspondences via vector measures.
\end{abstract}

(C) 2006 Elsevier B.V. All rights reserved.

\section{Introduction}

We introduce a new game form which allows the players' strategies to depend on their strategyrelevant private information as well as on some publicly announced information. The players' payoffs depend on their own payoff-relevant private information and some payoff-relevant common information. The purpose of this paper is to show that a pure strategy equilibrium exists for such game if the players' strategy-relevant private information is diffuse and their private information is conditionally independent given the public and payoff-relevant common information.

\footnotetext{
* Corresponding author.

E-mail addresses: fuhaifeng @nus.edu.sg(H.Fu), matsuny@nus.edu.sg (Y. Sun), nyanneli@uiuc.edu (N.C. Yannelis), zzx@jhu.edu (Z. Zhang)..
} 
The proof of the existence of a pure strategy equilibrium in our setting is far from trivial and requires the use of some new mathematical techniques. In particular, we develop a distribution theory of correspondences via vector measures that involves convexity, compactness and preservation of upper semi-continuity. This type of results allows us to apply Kakutani's fixed point theorem to prove the existence result based only on pure strategies. As noted in Khan and Sun (1995, p. 637), such a direct proof on the existence of a pure strategy equilibrium using only pure strategies does have some advantages from a game-theoretic point of view. In particular, one does not need to go through mixed (or behavioral) strategies that are considered to have limited appeal in many practical situations.

The paper is organized as follows. In Section 2, we introduce the game with private and public information and state the existence of a pure strategy equilibrium for such a game. Section 3 contains the main mathematical tool that is needed for our existence proof. Section 4 contains some concluding remarks. All the proofs are given in the appendix.

\section{Games with private and public information}

Consider a game $\Gamma$ with private and public information formulated as follows. The game has finitely many players $i=1, \ldots, l$. Each player $i$ is endowed with a finite action set $A_{i}$, a measurable space $\left(T_{i}, \mathcal{T}_{i}\right)$ representing her strategy-relevant private information, and another measurable space $\left(S_{i}, \mathcal{S}_{i}\right)$ representing her payoff-relevant private information. A finite set $T_{0}=$ $\left\{t_{01}, \ldots, t_{0 m}\right\}$ represents those states that are to be publicly announced to all the players; let $\mathcal{T}_{0}$ be the power set on $T_{0}$. Another finite set $S_{0}=\left\{s_{01}, \ldots, s_{0 n}\right\}$ represents the payoff-relevant common states that affect the payoffs of all the players with $\mathcal{S}_{0}$ the power set on $S_{0}$. Thus, the product measurable space $(\Omega, \mathcal{F})=\left(\Pi_{j=0}^{l}\left(T_{j} \times S_{j}\right), \Pi_{j=0}^{l}\left(\mathcal{T}_{j} \times \mathcal{S}_{j}\right)\right)$ equipped with a probability measure $\eta$ constitutes the information space of the game. For each player $i$, her payoff function is a mapping from $A \times S_{0} \times S_{i}$ to $R$, i.e. $u_{i}: A \times S_{0} \times S_{i} \longrightarrow R$. Here $A=\Pi_{j=1}^{l} A_{j}$ is the set of the players' action profiles; and assume that for any $a \in A, u_{i}\left(a, s_{0}, s_{i}\right)$ is integrable on $(\Omega, \mathcal{F}, \eta)$.

For each player $i$, she can use her private information as well as the publicly announced information. Thus, a pure strategy for player $i$ is a measurable mapping from $T_{0} \times T_{i}$ to $A_{i}$; and let Meas $\left(T_{0} \times T_{i}, A_{i}\right)$ be the space of all measurable mappings from $T_{0} \times T_{i}$ to $A_{i}$. A pure strategy profile is a collection $g=\left(g_{1}, \ldots, g_{l}\right)$ of pure strategies that specify a pure strategy for each player. For a player $i=1, \ldots, l$, we shall use the following (conventional) notation: $A_{-i}=\Pi_{1 \leq j \leq l, j \neq i} A_{j}, a=\left(a_{i}, a_{-i}\right)$ for $a \in A$, and $g=\left(g_{i}, g_{-i}\right)$ for a strategy profile $g .{ }^{1}$

To sum up, our game is of the form $\Gamma=\left\{A_{1}, \ldots, A_{l} ; T_{0} ; S_{0} ; T_{1}, \ldots, T_{l}\right.$; $\left.S_{1}, \ldots, S_{l} ; u_{1}, \ldots, u_{l}\right\}$, where $A_{1}, \ldots, A_{l}$ are the players' action spaces, $T_{0}$ is their public information space, $S_{0}$ is their payoff-relevant common information space, $T_{1}, \ldots, T_{l}$ are their strategyrelevant private information spaces, $S_{1}, \ldots, S_{l}$ are their payoff-relevant private information spaces and $u_{1}, \ldots, u_{l}$ are their payoff functions.

If the players play a pure strategy profile $g=\left(g_{1}, \ldots, g_{l}\right)$, the resulting expected payoff for player $i$ can be written as

$$
U_{i}(g)=U_{i}\left(g_{1}, \ldots, g_{l}\right)=\int_{\Omega} u_{i}\left(g_{1}\left(t_{0}, t_{1}\right), \ldots, g_{l}\left(t_{0}, t_{l}\right), s_{0}, s_{i}\right) \mathrm{d} \eta .
$$

A pure strategy equilibrium for $\Gamma$ is a pure strategy profile $g^{*}=\left(g_{1}^{*}, \ldots, g_{l}^{*}\right)$ such that for each $i=1, \ldots, l, g_{i}^{*}$ maximizes $U_{i}\left(g_{i}, g_{-i}^{*}\right)$ for $g_{i} \in \operatorname{Meas}\left(T_{0} \times T_{i}, A_{i}\right)$.

\footnotetext{
${ }^{1}$ From now on, without any ambiguity, we shall abbreviate $\Pi_{1 \leq j \leq l, j \neq i}$ to $\Pi_{j \neq i}$.
} 
The marginal measure of $\eta$ on $\left(T_{0} \times S_{0}, \mathcal{T}_{0} \times \mathcal{S}_{0}\right)$ is denoted by $\eta_{0}$. For simplicity, we denote $\eta_{0}\left(\left\{\left(t_{0 k}, s_{0 q}\right)\right\}\right)$ by $\alpha_{k q}$. For each given $t_{0 k} \in T_{0}$ and $s_{0 q} \in S_{0}$, let $\eta^{k q}$ denote the conditional probability measure of $\eta$ on the space $\left(\Pi_{j=1}^{l}\left(T_{j} \times S_{j}\right), \Pi_{j=1}^{l}\left(\mathcal{T}_{j} \times \mathcal{S}_{j}\right)\right)$. For each player $i=1, \ldots, l$, let $\tau_{i}$ be the marginal measure of $\eta$ on the space $\left(T_{i}, \mathcal{T}_{i}\right), \rho_{i}^{k q}$ the marginal measure of $\eta^{k q}$ on the space $\left(\left(T_{i} \times S_{i}\right) \times \Pi_{j \neq i} T_{j},\left(\mathcal{T}_{i} \times \mathcal{S}_{i}\right) \times \Pi_{j \neq i} \mathcal{T}_{j}\right), v_{i}^{k q}$ the marginal measure of $\eta^{k q}$ on the space $\left(T_{i} \times S_{i}, \mathcal{T}_{i} \times \mathcal{S}_{i}\right)$, and $\mu_{i}^{k q}$ be the marginal measure of $\eta^{k q}$ on the space $\left(T_{i}, \mathcal{T}_{i}\right)$.

\section{Definition 1.}

(1) The players' strategy-relevant private information is said to be diffuse if the marginal measure $\tau_{i}$ of $\eta$ on the space $\left(T_{i}, \mathcal{T}_{i}\right)$ is atomless for each player $i=1, \ldots, l$.

(2) The players' private information is said to be conditionally independent given the public and payoff-relevant common information if for each player $i=1, \ldots, l$, her strategy and payoff-relevant information is conditionally independent of all other players' strategy-relevant information, given $t_{0} \in T_{0}$ and $s_{0} \in S_{0}$. That is, $\rho_{i}^{k q}=v_{i}^{k q} \times \Pi_{j \neq i} \mu_{j}^{k q}$ for $k=1, \ldots, m$ and $q=1, \ldots, n$.

The following result shows the existence of a pure strategy equilibrium for the game $\Gamma$ under the assumption of diffuse and conditionally independent information.

Theorem 1. If the players' strategy-relevant private information is diffuse and their private information is conditionally independent given the public and payoff-relevant common information, then there exists a pure strategy equilibrium for the game $\Gamma$.

Independent payoff-relevant and strategy-relevant private information is used in the game studied in Radner and Rosenthal (1982). Milgrom and Weber (1985) consider games with payoffrelevant common information and private information that influences players' strategies and payoffs. ${ }^{2}$ Our model introduces the new concept of public information that influences all players' strategies, in addition to payoff-relevant and strategy-relevant private information and payoffrelevant common information. It is obvious that the existence results of pure strategy equilibrium in Milgrom and Weber (1985) and Radner and Rosenthal (1982) are special cases of our Theorem $1 .^{3}$

\section{Distribution of correspondences via vector measures}

In this section we present some properties of the distribution of correspondences induced by vector measures, which will be used to prove Theorem 1 . We recall some basic notions first.

Let $\Omega$ and $X$ be nonempty sets, and $\mathcal{P}(X)$ the power set of $X$. A mapping from $\Omega$ to $\mathcal{P}(X) \backslash\{\emptyset\}$ is called a correspondence from $\Omega$ to $X$.

Let $F$ be a correspondence from a measurable space $(\Omega, \mathcal{F})$ to a complete separable metric space $X$ with its Borel $\sigma$-algebra $\mathcal{B}(X)$, where $\mathcal{F}$ is a $\sigma$-algebra on $\Omega$. The correspondence $F$

\footnotetext{
${ }^{2}$ See Khan et al. (2006) for a unified approach to the purification of mixed strategies by using a consequence of the Dvoretzky-Wald-Wolfowitz Theorem in Dvoretzky et al. (1951).

3 The existence result of a pure strategy equilibrium in Milgrom and Weber (1985) is stated as a consequence of purification. However, the purification result in Milgrom and Weber (1985) does not follow directly from the original result in Dvoretzky et al. (1951) as claimed therein, but from a new corollary of the Dvoretzky-Wald-Wolfowitz Theorem formulated in Khan et al. (2006), where a stronger result on purification is also proved.
} 
is said to be measurable if for each closed subset $C$ of $X$, the set $\{\omega \in \Omega: F(\omega) \cap C \neq \emptyset\}$ is measurable in $\mathcal{F}$. The correspondence $F$ is said to be closed valued if $F(\omega)$ is a closed subset of $X$ for each $\omega \in \Omega$. A function $f$ from $(\Omega, \mathcal{F})$ to $X$ is said to be a measurable selection of $F$ if $f$ is measurable and $f(\omega) \in F(\omega)$ for all $\omega \in \Omega$. When $F$ is measurable and closed valued, the classical Kuratowski-Ryll-Nardzewski Theorem (see, for example, Aliprantis and Border (1994, p. 505)) says that $F$ has a measurable selection.

Let $\mathcal{M}(X)$ be the space of Borel probability measures on $X$ endowed with the topology of weak convergence of measures. Let $v$ be a probability measure and $\mu=\left(\mu_{1}, \ldots, \mu_{m}\right)$ a vector measure on $(\Omega, \mathcal{F})$, where each $\mu_{k}$ is a probability measure for $k=1, \ldots, m .(\Omega, \mathcal{F}, \mu)$ is called a vector probability measure space. For a measurable mapping $\varphi$ from a probability space $(\Omega, \mathcal{F}, v)$ to $X$, we use $\nu \varphi^{-1}$ to denote the Borel probability measure on $X$ induced by $\varphi$, which is often called the distribution of $\varphi$. We also use $\mu \varphi^{-1}$ to denote $\left(\mu_{1} \varphi^{-1}, \ldots, \mu_{m} \varphi^{-1}\right)$, which belongs to $(\mathcal{M}(X))^{m}$. When $X$ is a finite set $\left\{x_{1}, \ldots, x_{d}\right\}, \mathcal{M}(X)$ can be identified with the simplex $\Delta=\left\{\left(x_{1}, \ldots, x_{d}\right): x_{i} \geq 0, \sum_{i=1}^{d} x_{i}=1\right\}$ under the Euclidean metric.

Next, let $G$ be a correspondence from a topological space $Y$ to another topological space $Z$. Let $y_{0}$ be a point in $Y$. Then $G$ is said to be upper semicontinuous at $y_{0}$ if for any open set $U$ which contains $G\left(y_{0}\right)$, there exists a neighborhood $V$ of $y_{0}$ such that $y \in V$ implies that $G(y) \subseteq U$. $G$ is said to be upper semicontinuous on $Y$ if it is upper semicontinuous at every point $y \in Y$.

Now we state our main result about the distribution of correspondences induced by a vector measure when the target space is a finite set.

Proposition 1. Let $A$ be a finite set, $Y$ a metric space, $(\Omega, \mathcal{F}, \mu)$ an atomless vector probability measure space, ${ }^{4}$ and $F$ a correspondence from $\Omega \times Y$ to A. For each fixed $y \in Y$, let $F_{y}$ denote the correspondence $F(\cdot, y)$ from $\Omega$ to $A$, which is assumed to be measurable. Let $G$ be a correspondence from $Y$ to $(\mathcal{M}(A))^{m}$ such that for each $y \in Y$,

$$
G(y)=\left\{\mu \varphi^{-1}: \varphi(\cdot) \text { is a measurable selection from } F_{y}(\cdot)\right\} .
$$

Then, (1) $G$ is convex and compact valued; (2) if, in addition, the correspondence $F(\omega, \cdot)$ is upper semicontinuous on $Y$ for each fixed $\omega \in \Omega$, then $G$ is upper semicontinuous on $Y$.

Consider the simple case that $\mu$ is a scalar probability measure (i.e., $m=1$ ). All the three properties of convexity, compactness, and preservation of upper semicontinuity in the above theorem on the distribution of correspondences may fail when $A$ is not assumed to be finite (see Examples 1, 2 and 3 in Sun (1996) for the case that $A=[-1,1])$.

\section{Concluding remarks}

The game introduced in this paper can be easily extended to a social system by including constraint correspondences where action sets depend on the information of individual players. Such a framework may be useful to applications for economies with private information and also public information (see, for example Glycopantis and Yannelis, 2005). Thus, the standard Walrasian expectation equilibrium notions may be generalized by including the public information aspect as used in this paper.

\footnotetext{
${ }^{4}$ It means that $\mu_{k}$ is atomless for each $1 \leq k \leq m$.
} 


\section{Appendix A}

Proof of Theorem 1. First fix $i=1, \ldots, l$. Denote $g_{i}\left(t_{0 k}, t_{i}\right)$ by $g_{i}^{k}\left(t_{i}\right)$ for $k=1, \ldots, m$. Thus, for each $k, g_{i}^{k}$ is a mapping from $T_{i}$ to $A_{i}$. With the assumption of conditional independence in Theorem 1, we can rewrite player $i$ 's payoff in Eq. (1) as

$$
\begin{aligned}
U_{i}(g) & =\sum_{k=1}^{m} \sum_{q=1}^{n} \alpha_{k q} \int_{\Pi_{j=1}^{l}\left(T_{j} \times S_{j}\right)} u_{i}\left(g_{1}^{k}\left(t_{1}\right), \ldots, g_{l}^{k}\left(t_{l}\right), s_{0 q}, s_{i}\right) \mathrm{d} \eta^{k q} \\
& =\sum_{k=1}^{m} \sum_{q=1}^{n} \alpha_{k q} \int_{\left(T_{i} \times S_{i}\right) \times \Pi_{j \neq i} T_{j}} u_{i}\left(g_{1}^{k}\left(t_{1}\right), \ldots, g_{l}^{k}\left(t_{l}\right), s_{0 q}, s_{i}\right) \mathrm{d} \rho_{i}^{k q} \\
& =\sum_{k=1}^{m} \sum_{q=1}^{n} \alpha_{k q} \int_{\left(T_{i} \times S_{i}\right) \times \Pi_{j \neq i} T_{j}} u_{i}\left(g_{1}^{k}\left(t_{1}\right), \ldots, g_{l}^{k}\left(t_{l}\right), s_{0 q}, s_{i}\right) \mathrm{d}\left(v_{i}^{k q} \times \Pi_{j \neq i} \mu_{j}^{k q}\right) \\
& =\sum_{k=1}^{m} \sum_{q=1}^{n} \alpha_{k q} \int_{\left(T_{i} \times S_{i}\right) \times A_{-i}} u_{i}\left(g_{i}^{k}\left(t_{i}\right), a_{-i}, s_{0 q}, s_{i}\right) \mathrm{d}\left(v_{i}^{k q} \times \Pi_{j \neq i} \mu_{j}^{k q}\left(g_{j}^{k}\right)^{-1}\right) \\
& =\sum_{k=1}^{m} \sum_{q=1}^{n} \alpha_{k q} \int_{A_{-i}} \int_{T_{i} \times S_{i}} u_{i}\left(g_{i}^{k}\left(t_{i}\right), a_{-i}, s_{0 q}, s_{i}\right) \mathrm{d} v_{i}^{k q} \mathrm{~d} \Pi_{j \neq i} \mu_{j}^{k q}\left(g_{j}^{k}\right)^{-1} .
\end{aligned}
$$

Now we define the conditional expectation

$$
v_{i}^{k q}\left(a, t_{i}\right)=E\left(u_{i}\left(a, s_{0 q}, \tilde{s}_{i}\right) \mid \tilde{t}_{i}=t_{i}\right)
$$

where $\tilde{t}_{i}$ and $\tilde{s}_{i}$ are the projections from $\left(T_{i} \times S_{i}, \mathcal{T}_{i} \times \mathcal{S}_{i}, v_{i}^{k q}\right)$ to $T_{i}$ and $S_{i}$, respectively. Then,

$$
\int_{T_{i} \times S_{i}} u_{i}\left(g_{i}^{k}\left(t_{i}\right), a_{-i}, s_{0 q}, s_{i}\right) \mathrm{d} v_{i}^{k q}=\int_{T_{i}} v_{i}^{k q}\left(g_{i}^{k}\left(t_{i}\right), a_{-i}, t_{i}\right) \mathrm{d} \mu_{i}^{k q} .
$$

Thus, by substituting (4) into (3), we have

$$
\begin{aligned}
U_{i}(g) & =\sum_{k=1}^{m} \sum_{q=1}^{n} \alpha_{k q} \int_{A_{-i}} \int_{T_{i}} v_{i}^{k q}\left(g_{i}^{k}\left(t_{i}\right), a_{-i}, t_{i}\right) \mathrm{d} \mu_{i}^{k q} \mathrm{~d} \Pi_{j \neq i} \mu_{j}^{k q}\left(g_{j}^{k}\right)^{-1} \\
& =\sum_{k=1}^{m} \sum_{q=1}^{n} \int_{T_{i}} \alpha_{k q} \int_{A_{-i}} v_{i}^{k q}\left(g_{i}^{k}\left(t_{i}\right), a_{-i}, t_{i}\right) \mathrm{d} \Pi_{j \neq i} \mu_{j}^{k q}\left(g_{j}^{k}\right)^{-1} \mathrm{~d} \mu_{i}^{k q}=\sum_{k=1}^{m} V_{i}^{k}\left(g_{i}^{k}\right),
\end{aligned}
$$

where we write

$$
V_{i}^{k}\left(g_{i}^{k}\right)=\sum_{q=1}^{n} \int_{T_{i}} \alpha_{k q} \int_{A_{-i}} v_{i}^{k q}\left(g_{i}^{k}\left(t_{i}\right), a_{-i}, t_{i}\right) \mathrm{d} \Pi_{j \neq i} \mu_{j}^{k q}\left(g_{j}^{k}\right)^{-1} \mathrm{~d} \mu_{i}^{k q} .
$$

It means that player $i$ 's expected payoff depends on the actions of the other players only through the conditional distributions of their strategies (given the payoff-relevant common information $s_{0 q}$ and public information $t_{0 k}$ ) induced on their action spaces. Recall that the marginal measure $\tau_{i}$ of $\eta$ on the space $\left(T_{i}, \mathcal{T}_{i}\right)$ is atomless. This implies that if $\alpha_{k q}>0, \mu_{i}^{k q}$ is also atomless. When 
$\alpha_{k q}=0$, we can redefine $\mu_{i}^{k q}$ to be $\tau_{i}$ without changing anything. Thus, we can assume that $\mu_{i}^{k q}$ is also atomless for each $k=1, \ldots, m$, and $q=1, \ldots, n$.

Now fix $k \in\{1, \ldots, m\}$. Let $\lambda_{i}^{k}=\sum_{q=1}^{n} \mu_{i}^{k q}$. It is obvious to see that $\mathrm{d} \mu_{i}^{k q} / \mathrm{d} \lambda_{i}^{k}$ is well-defined with value in $[0,1]$ for each $i, k$ and $q$, and that $\sum_{q=1}^{n} \frac{\mathrm{d} \mu_{i}^{k q}}{\mathrm{~d} \lambda_{i}^{k}}\left(t_{i}\right)=1$ for $\lambda_{i}^{k}$-almost all $t_{i}$. For a given $\gamma \in \Pi_{j=1}^{l}\left[\left(\mathcal{M}\left(A_{j}\right)\right)^{n}\right],{ }^{5}$ Eq. (5) says that for each state of public information $t_{0}=t_{0 k}, k=$ $1, \ldots, m$, player $i$ should choose a response function $g_{i}^{k}: T_{i} \rightarrow A_{i}$ to maximize ${ }^{6}$

$$
\begin{aligned}
V_{i}^{k \gamma}\left(g_{i}^{k}\right) & =\sum_{q=1}^{n} \int_{T_{i}} \alpha_{k q} \int_{A_{-i}} v_{i}^{k q}\left(g_{i}^{k}\left(t_{i}\right), a_{-i}, t_{i}\right) \mathrm{d} \Pi_{j \neq i} \gamma_{j}^{q}\left(a_{-i}\right) \mathrm{d} \mu_{i}^{k q}\left(t_{i}\right) \\
& =\int_{T_{i}} \sum_{q=1}^{n} \alpha_{k q} \frac{\mathrm{d} \mu_{i}^{k q}}{\mathrm{~d} \lambda_{i}^{k}} \int_{A_{-i}} v_{i}^{k q}\left(g_{i}^{k}\left(t_{i}\right), a_{-i}, t_{i}\right) \mathrm{d} \Pi_{j \neq i} \gamma_{j}^{q}\left(a_{-i}\right) \mathrm{d} \lambda_{i}^{k} \\
& =\int_{T_{i}} w_{i}^{k}\left(g_{i}^{k}\left(t_{i}\right), t_{i}, \gamma\right) \mathrm{d} \lambda_{i}^{k}\left(t_{i}\right)
\end{aligned}
$$

where

$$
w_{i}^{k}\left(a_{i}, t_{i}, \gamma\right)=\sum_{q=1}^{n} \alpha_{k q} \frac{\mathrm{d} \mu_{i}^{k q}}{\mathrm{~d} \lambda_{i}^{k}}\left(t_{i}\right) \int_{A_{-i}} v_{i}^{k q}\left(a_{i}, a_{-i}, t_{i}\right) \mathrm{d} \Pi_{j \neq i} \gamma_{j}^{q}\left(a_{-i}\right) .
$$

It is obvious that for each $a_{i}$ in the finite set $A_{i}, w_{i}^{k}\left(a_{i}, t_{i}, \gamma\right)$ is $\mathcal{T}_{i}$-measurable with respect to $t_{i} \in T_{i}$, and continuous with respect to $\gamma \in \Pi_{j=1}^{l}\left[\left(\mathcal{M}\left(A_{j}\right)\right)^{n}\right]$.

For a fixed $k \in\{1, \ldots, m\}$ and for any $\gamma \in \Pi_{j=1}^{l}\left[\left(\mathcal{M}\left(A_{j}\right)\right)^{n}\right], t_{i} \in T_{i}$, let

$$
\Phi_{i}^{k}\left(t_{i}, \gamma\right)=\operatorname{Arg} \max _{a_{i} \in A_{i}} w_{i}^{k}\left(a_{i}, t_{i}, \gamma\right) .
$$

For each fixed $t_{i} \in T_{i}$, the correspondence $\Phi_{i}^{k}\left(t_{i}, \cdot\right)$ is upper semicontinuous on $\Pi_{j=1}^{l}\left[\left(\mathcal{M}\left(A_{j}\right)\right)^{n}\right]$ by Berge's maximum theorem (see, for example, Aliprantis and Border (1994, p. 473)). For any fixed $\gamma \in \Pi_{j=1}^{l}\left[\left(\mathcal{M}\left(A_{j}\right)\right)^{n}\right]$, the correspondence $\Phi_{i}^{k}(\cdot, \gamma)$ is measurable by Theorem 14.91 in Aliprantis and Border (1994, p. 508).

Consider a pure strategy profile $g^{*}=\left(g_{1}^{*}, \ldots, g_{l}^{*}\right)$ and let $g^{* k}=\left(g_{1}^{* k}, \ldots, g_{l}^{* k}\right)$ be defined as $g_{i}^{* k}(\cdot)=g_{i}^{*}\left(t_{0 k} ; \cdot\right), k=1, \ldots, m$.

Denote the $n$-dimensional vector measure $\left(\mu_{i}^{k 1}, \ldots, \mu_{i}^{k n}\right)$ by $\mu_{i}^{k}$. Let $\gamma_{i}^{* k}$ be the conditional distribution $\mu_{i}^{k}\left(g_{i}^{* k}\right)^{-1}$ of player $i$, and $\gamma^{* k}=\left(\gamma_{1}^{* k}, \ldots, \gamma_{l}^{* k}\right)$. Then, $g^{*}$ is a pure strategy equilibrium for the game $\Gamma$ if and only if for each player $i$ and each $k, g_{i}^{* k}$ maximizes $V_{i}^{k \gamma^{* k}}(\cdot)$ on the space Meas $\left(T_{i}, A_{i}\right){ }^{7}$ This condition can be satisfied if $g_{i}^{* k}$ is a measurable selection of the correspondence $\Phi_{i}^{k}\left(\cdot, \gamma^{* k}\right)$ for any $k$ and $i$.

\footnotetext{
${ }^{5}$ For any player $j$, let $\gamma_{j}=\left(\gamma_{j}^{1}, \ldots, \gamma_{j}^{n}\right) \in\left(\mathcal{M}\left(A_{j}\right)\right)^{n}$, which can be interpreted as a conditional distribution for player $j$ 's strategy given the payoff-relevant common information $s_{0 q}$ and public information $t_{0 k}$. Let $\gamma=\left(\gamma_{1}, \ldots, \gamma_{l}\right)$ which specifies a conditional distribution for each player while $\gamma_{-i}$ specifies the conditional distributions for all the players except for player $i$.

${ }^{6}$ The function $V_{i}^{k \gamma}\left(g_{i}^{k}\right)$ is actually independent of $\gamma_{i}$. However, it is more convenient, as we do, to take the whole $\gamma$ as a parameter.

7 The space of all measurable mappings from $T_{i}$ to $A_{i}$.
} 
We shall now show the existence of such a pure strategy profile $g^{*}$. For any $\gamma \in$ $\Pi_{j=1}^{l}\left[\left(\mathcal{M}\left(A_{j}\right)\right)^{n}\right]$ and any $k \in\{1, \ldots, m\}$, let

$$
G_{i}^{k}(\gamma)=\left\{\mu_{i}^{k} \varphi^{-1}: \varphi(\cdot) \text { is a measurable selection from } \Phi_{i}^{k}(\cdot, \gamma)\right\},
$$

and $G^{k}(\gamma)=\Pi_{i=1}^{l} G_{i}^{k}(\gamma)$. Proposition 1 implies that for each $i, G_{i}^{k}(\cdot)$ is convex and compact valued, and upper-semicontinuous on $\Pi_{j=1}^{l}\left[\left(\mathcal{M}\left(A_{j}\right)\right)^{n}\right]$, so is the product $G^{k}(\gamma)$. By the Kakutani Fixed Point Theorem, there exists a $\gamma^{* k}=\left(\gamma_{1}^{* k}, \ldots, \gamma_{l}^{* k}\right) \in \Pi_{j=1}^{l}\left[\left(\mathcal{M}\left(A_{j}\right)\right)^{n}\right]$ such that $\gamma^{* k} \in G^{k}\left(\gamma^{* k}\right)$. This means that for each player $i$ and each $k, \gamma_{i}^{* k} \in G_{i}^{k}\left(\gamma^{* k}\right)$, i.e., there exists a measurable selection $g_{i}^{* k}$ of the correspondence $\Phi_{i}^{k}\left(\cdot, \gamma^{* k}\right)$ such that $\mu_{i}^{k}\left(g_{i}^{* k}\right)^{-1}=\gamma_{i}^{* k}$. Therefore, the pure strategy profile $g^{*}=\left(g_{1}^{*}, \ldots, g_{l}^{*}\right)$ is a pure strategy equilibrium for the game $\Gamma$.

To prove Proposition 1, we need part of Corollary 1 in Khan et al. (2006), which is presented in the following lemma for the convenience of the reader. The result is a simple consequence of Theorem 2.1 in Dvoretzky et al. (1951).

Lemma A.1. Let $(\Omega, \mathcal{F}, \mu)$ be an atomless vector probability measure space with $\mu=$ $\left(\mu_{1}, \ldots, \mu_{m}\right), A$ a finite set with elements $a_{1}, \ldots, a_{d}$, and $g: \Omega \rightarrow \mathcal{M}(A)$ a measurable mapping. Then there exists a measurable mapping $g^{*}: \Omega \rightarrow$ A such that $g^{*}(\omega) \in \operatorname{supp} g(\omega)=\{a \in$ $A: g(\omega)(\{a\})>0\}$ for all $\omega \in \Omega$, and for each $a \in A, \int_{\Omega} g(\omega)(\{a\}) \mathrm{d} \mu(\omega)=\mu g^{*-1}(\{a\})$.

Let $A_{n}, n=1,2, \ldots$ be a sequence of sets in a metric space $X$. A point $x \in X$ is said to be a cluster point of the sequence of sets if every neighborhood of $x$ intersects infinitely many $A_{n}$. The set of all such cluster points is denoted by $c l-\operatorname{Lim}_{n} A_{n}$, which is also called topological limes superior (see, for example, Definition 3.10 in Sun (1996)). Note that when $X$ is a finite set, one can use any metric introducing the discrete topology.

Before proving Proposition 1, we prove two more lemmas.

Lemma A.2. Let $f=\left(f_{1}, \ldots, f_{d}\right)$ and $f^{n}=\left(f_{1}^{n}, \ldots, f_{d}^{n}\right), n=1,2, \ldots$, be measurable functions from a probability space $(\Omega, \mathcal{F}, v)$ to the unit simplex $\Delta=\left\{\left(x_{1}, \ldots, x_{d}\right): x_{i} \geq 0, \sum_{i=1}^{d} x_{i}=\right.$ $1\}$. Assume that for each $k \in\{1, \ldots, d\}, f_{k}^{n}$ converges to $f_{k}$ in the weak star topology $\sigma\left(L^{\infty}(\Omega), L^{1}(\Omega)\right)$. Then, for v-almost all $\omega \in \Omega,\left\{k \in\{1, \ldots, d\}: f_{k}(\omega)>0\right\} \subseteq$ cl- $\operatorname{Lim}_{n}\{k \in$ $\left.\{1, \ldots, d\}: f_{k}^{n}(\omega)>0\right\}$.

Proof. Suppose not. Then there exists a measurable subset $E \subseteq \Omega$ of positive measure with respect to $\nu$, with the following property: for all $\omega \in E$, the inclusion relation $\left\{k^{\prime} \in\{1, \ldots, d\}: f_{k^{\prime}}(\omega)>0\right\} \subseteq c l-\operatorname{Lim}_{n}\left\{k^{\prime} \in\{1, \ldots, d\}: f_{k^{\prime}}^{n}(\omega)>0\right\}$ fails. So there exists a $k \in\{1, \ldots, d\}$ and a set $E_{k} \subseteq E$ with $v$-positive measure, such that for any $\omega \in$ $E_{k}, f_{k}(\omega)>0$, and $k \notin c l-\operatorname{Lim}_{n}\left\{k^{\prime} \in\{1, \ldots, d\}: f_{k^{\prime}}^{n}(\omega)>0\right\}$, which means that $k \notin\left\{k^{\prime} \in\right.$ $\left.\{1, \ldots, d\}: f_{k^{\prime}}^{n}(\omega)>0\right\}$ for sufficiently large $n$. Thus, for any $\omega \in E_{k}, f_{k}^{n}(\omega)=0$ for sufficiently large $n$.

Let $1_{E_{k}}$ be the indicator function of $E_{k}$. Note that $f_{k}^{n}$ is assumed to converge to $f_{k}$ in the weak star topology $\sigma\left(L^{\infty}(\Omega), L^{1}(\Omega)\right)$. This implies that

$$
\lim _{n \rightarrow \infty} \int_{\Omega} f_{k}^{n}(\omega) 1_{E_{k}}(\omega) \mathrm{d} v(\omega)=\int_{\Omega} f_{k}(\omega) 1_{E_{k}}(\omega) \mathrm{d} \nu(\omega)
$$

We observe that $\int_{\Omega} f_{k}^{n}(\omega) 1_{E_{k}}(\omega) \mathrm{d} \nu(\omega)=\int_{E_{k}} f_{k}^{n}(\omega) \mathrm{d} \nu(\omega)$, which equals zero for sufficiently large $n$, and thus the left hand side of Eq. (10) is zero. On the other hand, the right hand side of Eq. 
(10) is $\int_{\Omega} f_{k}(\omega) 1_{E_{k}}(\omega) \mathrm{d} \nu(\omega)=\int_{E_{k}} f_{k}(\omega) \mathrm{d} \nu(\omega)$, which is strictly positive since $\nu\left(E_{k}\right)>0$, and $f_{k}(\omega)>0$ for any $\omega \in E_{k}$. This is a contradiction.

Next we turn to Lemma A.3.

Lemma A.3. Let $(\Omega, \mathcal{F}, \mu)$ be an atomless vector probability measure space with $\mu=$ $\left(\mu_{1}, \ldots, \mu_{m}\right), A=\left\{a_{1}, \ldots, a_{d}\right\}$, and $\varphi_{n}, n=1,2, \ldots$ a sequence of measurable functions from $(\Omega, \mathcal{F})$ to $A$. Let $\mu \varphi_{n}^{-1}=\left(\mu_{1} \varphi_{n}^{-1}, \ldots, \mu_{m} \varphi_{n}^{-1}\right)$ for $n \geq 1$, and $\tau=\left(\tau_{1}, \ldots, \tau_{m}\right)$ a vector of probability measures on $A$. Assume that for each $k=1, \ldots, m$, and each $a \in A, \mu_{k}\left(\varphi_{n}^{-1}(\{a\})\right)$ converges to $\tau_{k}(\{a\})$ as $n$ goes to infinity. Then there exists a measurable selection $\varphi$ of the correspondence $H=c l-\operatorname{Lim}_{n}\left\{\varphi_{n}\right\}$ such that $\mu \varphi^{-1}=\tau$.

Proof. Since $A$ is finite, the classical Alaoglu Compactness Theorem (see, for example, Aliprantis and Border (1994, p. 158)) implies that there exists a subsequence $\varphi_{n_{q}}, q=1,2, \ldots$ of $\varphi_{n}, n=$ $1,2, \ldots$ such that for each $a \in A,\left\{1_{\{a\}}\left(\varphi_{n_{q}}(\cdot)\right): q=1,2, \ldots\right\}$ converges to some function $f_{a}(\cdot) \in$ $L^{\infty}(\Omega, \mathcal{F},|\mu|)$ in the weak star topology $\sigma\left(L^{\infty}(\Omega, \mathcal{F},|\mu|), L^{1}(\Omega, \mathcal{F},|\mu|)\right)$, where $|\mu|$ denotes the probability measure $(1 / m) \sum_{i=1}^{m} \mu_{i}$.

For each $q \geq 1$ and each $a \in A$, let $f_{a}^{q}(\cdot)=1_{\{a\}}\left(\varphi_{n_{q}}(\cdot)\right)$; then $f_{a}^{q}, a \in A$ are non-negative functions satisfying $\sum_{a \in A} f_{a}(\cdot) \equiv 1$. Since $f_{a}^{q}(\cdot)$ converges to $f_{a}(\cdot)$ in the weak star topology $\sigma\left(L^{\infty}(\Omega, \mathcal{F},|\mu|), L^{1}(\Omega, \mathcal{F},|\mu|)\right)$ as $q$ goes to infinity, $f_{a}(\omega), a \in A$ are non-negative with summation one for $|\mu|$-almost all $\omega \in \Omega$; we can assume, without loss of generality, that this property holds for all $\omega \in \Omega$.

By the convergence assumption in the statement of the lemma, we have

$$
\int_{\Omega} f_{a}^{q}(\omega) \mathrm{d} \mu_{k}=\mu_{k}\left(\varphi_{n_{q}}^{-1}(\{a\})\right),
$$

which converges to $\tau_{k}(\{a\})$ as $q$ goes to infinity. Let $\mathrm{d} \mu_{k} / \mathrm{d}|\mu|$ be the Radon-Nikodym derivative of $\mu_{k}$ with respect to $|\mu|$. Since $f_{a}^{q}(\cdot)$ converges to $f_{a}(\cdot)$ in the weak star topology $\sigma\left(L^{\infty}(\Omega, \mathcal{F},|\mu|), L^{1}(\Omega, \mathcal{F},|\mu|)\right)$ as $q$ goes to infinity, we have

$$
\lim _{q \rightarrow \infty} \int_{\Omega} f_{a}^{q}(\omega) \mathrm{d} \mu_{k}=\lim _{q \rightarrow \infty} \int_{\Omega} f_{a}^{q}(\omega) \frac{\mathrm{d} \mu_{k}}{\mathrm{~d}|\mu|} \mathrm{d}|\mu|=\int_{\Omega} f_{a}(\omega) \frac{\mathrm{d} \mu_{k}}{\mathrm{~d}|\mu|} \mathrm{d}|\mu| .
$$

Hence, we obtain that

$$
\tau_{k}(\{a\})=\int_{\Omega} f_{a}(\omega) \frac{\mathrm{d} \mu_{k}}{\mathrm{~d}|\mu|} \mathrm{d}|\mu|=\int_{\Omega} f_{a}(\omega) \mathrm{d} \mu_{k} .
$$

It follows from Lemma A.2 that for $|\mu|$-almost all $\omega \in \Omega$,

$$
\left\{a \in A: f_{a}(\omega)>0\right\} \subseteq c l-\operatorname{Lim}_{q}\left\{a \in A: f_{a}^{q}(\omega)>0\right\}=c l-\operatorname{Lim}_{q}\left\{\varphi_{n_{q}}(\omega)\right\} \subseteq c l-\operatorname{Lim}_{n}\left\{\varphi_{n}(\omega)\right\} .
$$

Define a mapping $g$ on $\Omega$ by letting $g(\omega)(\{a\})=f_{a}(\omega)$ for $\omega \in \Omega$ and $a \in A$. Then $g$ is a measurable mapping from $\Omega$ to $\mathcal{M}(A)$. Applying Lemma A.1 to $g$, we know that there exists a measurable mapping $\varphi: \Omega \rightarrow A$ such that $\varphi(\omega) \in\left\{a \in A: f_{a}(\omega)>0\right\}$, and $\mu \varphi^{-1}(\{a\})=\int_{\Omega} f_{a}(\omega) \mathrm{d} \mu(\omega)$. Hence, $\mu \varphi^{-1}=\tau$. Note that we also have $\varphi(\omega) \in c l-\operatorname{Lim}_{n}\left\{\varphi_{n}(\omega)\right\}$ for $|\mu|$-almost all $\omega \in \Omega$. By modifying the values of $\varphi$ on a $|\mu|$-null set through a measurable selection of $c l-\operatorname{Lim}_{n}\left\{\varphi_{n}(\cdot)\right\}$, we can require that $\varphi(\omega) \in c l-\operatorname{Lim}_{n}\left\{\varphi_{n}(\omega)\right\}$ for all $\omega \in \Omega$.

We are now ready to prove Proposition 1.

Proof of Proposition 1. Fix $y \in Y$. To prove the convexity, let $c \in[0,1]$ and $\varphi$ and $\tilde{\varphi}$ be two measurable selections from $F_{y}(\cdot)$. Let $\tau=c \mu \varphi^{-1}+(1-c) \mu \tilde{\varphi}^{-1}$. Define a mapping $f: \Omega \rightarrow$ 
$\mathcal{M}(A)$ by letting $f(\omega)=c \delta_{\varphi(\omega)}+(1-c) \delta_{\tilde{\varphi}(\omega)}$, where $\delta_{a}$ is the Dirac measure at $a$ for $a \in A$. Then $\tau(\{a\})=\int_{\Omega} f(\omega)(\{a\}) \mathrm{d} \mu(\omega)$ for any $a \in A$. By Lemma A.1, there exists a measurable function $\psi: \Omega \rightarrow A$ such that $\tau=\mu \psi^{-1}$ and $\psi(\omega) \in\{\varphi(\omega), \tilde{\varphi}(\omega)\}$ for all $\omega \in \Omega$. Since $\{\varphi(\omega), \tilde{\varphi}(\omega)\} \subseteq$ $F_{y}(\omega)$, this implies that $\psi$ is a measurable selection of $F_{y}$. Hence, $\tau \in G(y)$ and $G(y)$ is convex.

Now we turn to the upper semicontinuity of $G(\cdot)$, or equivalently, the closeness of the graph of correspondence $G(\cdot)$. Suppose that $y_{n}$ converges to $y$ in $Y, \tau_{n}=\mu \varphi_{n}^{-1} \in G\left(y_{n}\right)$ and $\tau_{n}$ converges to $\tau$, where $\varphi_{n}(\cdot) \in F_{y_{n}}(\cdot)$. We need only show that $\tau \in G(y)$. In fact, Lemma A.3 implies that there exists a measurable selection $\varphi$ of the correspondence $c l-\operatorname{Lim}_{n}\left\{\varphi_{n}\right\}$ such that $\mu \varphi^{-1}=\tau$. The upper-semicontinuity of $F_{y}$ with respect to $y$ implies the relation $c l-\operatorname{Lim}_{n}\left\{\varphi_{n}\right\} \subseteq F_{y}$. So $\varphi$ is a measurable selection of $F_{y}$, hence $\tau \in G(y)$. Thus, we obtain the upper-semicontinuity of the correspondence $G(\cdot)$.

Since $(\mathcal{M}(A))^{m}$ is compact, the compactness of $G(\cdot)$ follows from its closedness while the closedness follows from upper-semicontinuity by taking $y_{n}=y$ above.

\section{References}

Aliprantis, C.D., Border, K.C., 1994. Infinite dimensional analysis: a Hitchhiker's guide. Springer-Verlag, Berlin.

Dvoretzky, A., Wald, A., Wolfowitz, J., 1951. Elimination of randomization in certain statistical decision procedures and zero-sum two-person games. Ann. Math. Stat. 22, 1-21.

Glycopantis, D., Yannelis, N.C. (Eds.), 2005. Differential Information Economics, Studies in Economis Theory, vol. 19. Springer.

Khan, M.A., Rath, K.P., Sun, Y.N., 2006. The Dvoretzky-Wald-Wolfowitz theorem and purification in atomless finiteaction games. Int. J. Game Theory 34, 91-104.

Khan, M.A., Sun, Y.N., 1995. Pure strategies in games with private information. J. Math. Econ. 24, 633-653.

Milgrom, P.R., Weber, R.J., 1985. Distributional strategies for games with incomplete information. Math. Oper. Res. 10, 619-632.

Radner, R., Rosenthal, R.W., 1982. Private information and pure-strategy equilibria. Math. Oper. Res. 7, 401-409.

Sun, Y.N., 1996. Distributional properties of correspondences on Loeb spaces. J. Funct. Anal. 139, 68-93. 